\title{
Parameterization of single-scattering albedo (SSA) and absorption Ångström exponent (AAE) with EC / OC for aerosol emissions from biomass burning
}

Rudra P. Pokhrel et al.

Correspondence to: Shane M. Murphy (shane.murphy@uwyo.edu)

The copyright of individual parts of the supplement might differ from the CC-BY 3.0 licence. 
Table S1. Summary of fuels analyzed in this study and sampling locations.

\begin{tabular}{|l|l|l|l|l|}
\hline & $\begin{array}{l}\text { Stack } \\
\text { Burn }\end{array}$ & $\begin{array}{l}\text { Room } \\
\text { Burn }\end{array}$ & Fuel type & Sampling location \\
\hline $\begin{array}{l}\text { African grass } \\
\text { (tall) }\end{array}$ & 0 & 1 & Savana/Sourveld/Tall grass & Kruger National Park, R.S.A \\
\hline $\begin{array}{l}\text { African grass } \\
\text { (short) }\end{array}$ & 4 & 0 & Savana/Sourveld/Short grass & Kruger National Park, R.S.A \\
\hline Giant Cutgrass & 0 & 1 & Marsh & Jasper CO., SC \\
\hline Wiregrass & 0 & 1 & Pine forest understory & Chesterfield Co., SC \\
\hline Peat (CAN) & 2 & 0 & Boreal Peat & Ontario \& Alberta, Canada \\
\hline Peat (NC) & 2 & 1 & Temperate Peat & $\begin{array}{l}\text { Green Swamp \& Alligator River } \\
\text { NWR, NC }\end{array}$ \\
\hline Peat (IN) & 2 & 0 & Indonesian Peat & Central Kalimantan \\
\hline Organic Hay & 1 & 2 & Crop residue & Fort Collins, CO \\
\hline $\begin{array}{l}\text { Organic Wheat } \\
\text { Straw }\end{array}$ & 1 & 2 & Crop residue & Fort Collins, CO \\
\hline $\begin{array}{l}\text { Conv. Wheat } \\
\text { Straw }\end{array}$ & 0 & 1 & Crop residue & Walla Walla CO., WA \\
\hline Sugar Cane & 0 & 1 & Crop residue & Thibodaux, LA \\
\hline Rice Straw & 1 & 2 & Crop residue & CA, China, Malaysia, Taiwan \\
\hline Ponderosa Pine & 5 & 4 & Temperate Forest & Outskirts of Missoula, MT \\
\hline Black Spruce & 0 & 3 & Boreal Forest & South of Fairbanks, AK \\
\hline Chamise & 1 & 1 & Chaparral & San Jacinto Mountains, CA \\
\hline Manzanita & 1 & 1 & Chaparral & San Jacinto Mountains, CA \\
\hline Total & 20 & 21 & & \\
\hline & & & & \\
\hline
\end{tabular}


Table S2. SSA, AAE, MCE, EC to OC ratio, and mass of fuel burned for all fuels measured during stack and room burns of this work. S and $\mathrm{K}$ represent the short and tall African grass and the number after $\mathrm{S}$ or $\mathrm{K}$ indicate the collection plots. The \pm values after AAE are the one standard deviation of the slope and for EC/OC is the propagated error from EC and OC uncertainty. Errors for SSA are not shown because of space considerations, but are $\pm 7 \%$ of the quantity (1-SSA) for 532 and $660 \mathrm{~nm}$, whereas $\pm 18 \%$ of the quantity (1-SSA) for $405 \mathrm{~nm}$. For instance, the error for an SSA of 0.95 is \pm 0.004 while the error at an SSA of 0.5 is \pm 0.036 for 532 and $660 \mathrm{~nm}$.

\begin{tabular}{|c|c|c|c|c|c|c|c|c|}
\hline \multirow[b]{2}{*}{ ID } & \multirow[b]{2}{*}{ Fuel Type } & \multicolumn{3}{|c|}{ SSA } & \multirow[b]{2}{*}{$\mathbf{A A E}$} & \multirow[b]{2}{*}{ MCE } & \multirow[b]{2}{*}{ EC/OC } & \multirow[b]{2}{*}{ Mass (g) } \\
\hline & & 405 & 532 & 660 & & & & \\
\hline 9 & Manzanita & 0.79 & 0.8 & 0.79 & $2.05 \pm 0.37$ & 0.93 & NA & 270 \\
\hline 18 & Ponderosa Pine & 0.88 & 0.95 & 0.95 & $3.69 \pm 0.48$ & 0.918 & $0.062 \pm 0.018$ & 202 \\
\hline 20 & Chamise & 0.79 & 0.78 & 0.83 & $2.52 \pm 1.58$ & 0.913 & NA & 250 \\
\hline 32 & Rice Straw & 0.71 & 0.86 & 0.91 & $4.16 \pm 0.11$ & 0.943 & $0.111 \pm 0.028$ & 430 \\
\hline 61 & NC Peat & 0.94 & 1 & 1 & $10.43 \pm 1.11$ & 0.683 & ND & NA \\
\hline 62 & Ponderosa Pine & 0.83 & 0.91 & 0.89 & $2.85 \pm 0.48$ & 0.946 & $0.084 \pm 0.016$ & 4029 \\
\hline 70 & Ponderosa Pine & 0.93 & 0.99 & 0.99 & $5.95 \pm 0.79$ & 0.848 & $0.021 \pm 0.018$ & 274 \\
\hline 81 & African Grass S2 & 0.72 & 0.78 & 0.81 & $2.25 \pm 0.33$ & 0.97 & NA & 436 \\
\hline 82 & African Grass S1 & 0.53 & 0.55 & 0.54 & $1.92 \pm 0.27$ & 0.978 & NA & 416 \\
\hline 84 & Ponderosa Pine & 0.82 & 0.87 & 0.87 & $2.05 \pm 0.23$ & 0.925 & NA & 3860 \\
\hline 87 & Organic Hay & 0.81 & 0.91 & 0.94 & $3.46 \pm 0.08$ & 0.941 & NA & 412 \\
\hline 91 & African Grass S1 & 0.84 & 0.92 & 0.87 & $3.05 \pm 0.39$ & 0.97 & NA & 580 \\
\hline 92 & African Grass S2 & 0.58 & 0.62 & 0.58 & $1.72 \pm 0.08$ & 0.972 & NA & 455 \\
\hline 95 & Ponderosa Pine & 0.78 & 0.85 & 0.85 & $2.48 \pm 0.08$ & 0.933 & NA & 150 \\
\hline 96 & Organic Wheat & 0.73 & 0.76 & 0.87 & $3.43 \pm 1.13$ & 0.965 & $0.239 \pm 0.054$ & 154 \\
\hline 112 & Canadian Peat & 0.97 & 1 & 1 & $5.11 \pm 1.58$ & 0.811 & $0.011 \pm 0.012$ & NA \\
\hline 113 & NC Peat & 0.94 & 0.98 & 0.98 & $3.68 \pm 0.47$ & 0.692 & $0.008 \pm 0.007$ & NA \\
\hline 114 & Indonesian Peat & 0.93 & 1 & 0.99 & $7.44 \pm 3.27$ & 0.744 & $0.005 \pm 0.010$ & NA \\
\hline 124 & Canadian Peat & 0.91 & 0.99 & 0.99 & $6.78 \pm 0.26$ & 0.798 & NA & NA \\
\hline 125 & Indonesian Peat & 0.93 & 0.98 & 0.99 & $8.03 \pm 2.50$ & 0.872 & NA & NA \\
\hline 129 & $\begin{array}{l}\text { Ponderosa Pine } \\
\text { brown/green }\end{array}$ & 0.92 & 0.98 & 0.98 & $4.92 \pm 0.46$ & 0.839 & $0.068 \pm 0.018$ & 200 \\
\hline 130 & $\begin{array}{l}\text { California Rice } \\
\text { Straw }\end{array}$ & 0.75 & 0.84 & 0.84 & $2.98 \pm 0.20$ & 0.961 & $0.192 \pm 0.031$ & 147 \\
\hline 131 & Black Spruce & 0.87 & 0.93 & 0.93 & $2.98 \pm 0.05$ & 0.957 & $0.095 \pm 0.018$ & 639 \\
\hline 132 & Organic Wheat & 0.82 & 0.88 & 0.88 & $2.58 \pm 0.01$ & 0.956 & $0.127 \pm 0.024$ & 638 \\
\hline 133 & $\begin{array}{l}\text { Conventional } \\
\text { Wheat }\end{array}$ & 0.79 & 0.85 & 0.86 & $2.67 \pm 0.03$ & 0.956 & $0.150 \pm 0.023$ & 494 \\
\hline 134 & Black Spruce & 0.87 & 0.93 & 0.93 & $2.97 \pm 0.12$ & 0.957 & NA & 1077 \\
\hline 135 & Chamise & 0.58 & 0.61 & 0.54 & $1.95 \pm 0.06$ & 0.954 & NA & 667 \\
\hline 136 & Manzanita & 0.52 & 0.56 & 0.46 & $1.68 \pm 0.09$ & 0.959 & $0.711 \pm 0.060$ & 1064 \\
\hline 137 & Black Spruce & 0.82 & 0.9 & 0.84 & $2.32 \pm 0.70$ & 0.962 & NA & 1602 \\
\hline 138 & Organic Hay & 0.85 & 0.94 & 0.92 & $3.25 \pm 0.79$ & 0.95 & NA & 592.2 \\
\hline
\end{tabular}




\begin{tabular}{|l|l|l|l|l|l|l|l|l|}
140 & Ponderosa Pine & 0.88 & 0.94 & 0.91 & $2.21 \pm 0.61$ & 0.928 & $0.039 \pm 0.012$ & 1672 \\
\hline 141 & Wire Grass & 0.36 & 0.38 & 0.35 & $0.85 \pm 0.21$ & 0.969 & $1.386 \pm 0.113$ & 540 \\
\hline 142 & $\begin{array}{l}\text { Ponderosa Pine } \\
\text { brown/green }\end{array}$ & 0.79 & 0.83 & 0.82 & $2.19 \pm 0.19$ & 0.952 & NA & 1529 \\
\hline 143 & $\begin{array}{l}\text { California Rice } \\
\text { Straw }\end{array}$ & 0.87 & 0.95 & 0.97 & $4.48 \pm 0.05$ & 0.939 & NA & 902 \\
\hline 144 & Ponderosa Pine & 0.86 & 0.91 & 0.93 & $2.68 \pm 0.21$ & 0.927 & $0.037 \pm 0.013$ & 1731 \\
\hline 145 & African Grass K3 & 0.45 & 0.32 & 0.26 & $1.29 \pm 0.48$ & 0.975 & $1.206 \pm 0.097$ & 1078 \\
\hline 146 & Organic Hay & 0.85 & 0.94 & 0.96 & $3.55 \pm 0.08$ & 0.937 & $0.027 \pm 0.010$ & 1335 \\
\hline 147 & Sugarcane & 0.85 & 0.93 & 0.94 & $4.02 \pm 0.13$ & 0.934 & $0.052 \pm 0.021$ & 867 \\
\hline 148 & Giant Saw Grass & 0.39 & 0.43 & 0.44 & $1.83 \pm 0.34$ & 0.925 & $2.148 \pm 0.177$ & 2000 \\
\hline 149 & Organic Wheat & 0.77 & 0.83 & 0.83 & $2.58 \pm 0.08$ & 0.962 & $0.185 \pm 0.029$ & 393 \\
\hline 150 & $\begin{array}{l}\text { North Carolina } \\
\text { Peat }\end{array}$ & 0.95 & 0.99 & 1 & $6.25 \pm 0.63$ & 0.803 & NA & NA \\
\hline
\end{tabular}


Table S3. Same as Table 5 of main text, but for $405 \mathrm{~nm}$.

\begin{tabular}{|l|l|l|l|r|l|l|}
\hline Biomass Types & MCE & BC/OC & $\begin{array}{l}\text { SSA_405 } \\
\text { ACE } \\
\text { Approach }\end{array}$ & $\begin{array}{l}\text { SSA_405 } \\
\text { EC/OC } \\
\text { Approach }\end{array}$ & $\begin{array}{l}\text { SSA_405 } \\
\text { EC/EC+OC) } \\
\text { Approach }\end{array}$ & $\begin{array}{l}\text { \% Difference } \\
\text { In Predicted } \\
\text { SSA }\end{array}$ \\
\hline Tropical Forest & 0.95 & 0.11 & 0.74 & 0.81 & 0.83 & $-9 /-12$ \\
\hline Savanna & 0.96 & 0.14 & 0.63 & 0.78 & 0.80 & $-24 /-27$ \\
\hline Crop Residue & 0.94 & 0.33 & 0.77 & 0.69 & 0.70 & $10 / 9$ \\
\hline Pasture Maintenance & 0.92 & 0.09 & 0.84 & 0.82 & 0.84 & $2 / 0$ \\
\hline Boreal Forest & 0.92 & - & 0.84 & & - & - \\
\hline Temperate Forest & 0.95 & - & 0.73 & & - & - \\
\hline Extra tropical Forest & 0.93 & 0.07 & 0.83 & 0.85 & 0.86 & $-2 /-4$ \\
\hline Peat land & 0.9 & 0.03 & 0.90 & 0.89 & 0.89 & $1 / 1$ \\
\hline Chaparral & 0.96 & 0.35 & 0.64 & 0.68 & 0.68 & $-6 /-6$ \\
\hline Open Cooking & 0.95 & 0.29 & 0.70 & 0.71 & 0.72 & $-1 /-3$ \\
\hline Patsari Stoves & 0.97 & 0.39 & 0.54 & 0.67 & 0.67 & $-24 /-24$ \\
\hline Charcoal Making & 0.86 & 0.03 & 0.93 & 0.89 & 0.89 & $4 / 4$ \\
\hline Charcoal Burning & 0.93 & 0.77 & 0.82 & 0.55 & 0.53 & $33 / 35$ \\
\hline Dung Burning & 0.89 & 0.29 & 0.90 & 0.7 & 0.71 & $22 / 21$ \\
\hline Garbage Burning & 0.97 & 0.12 & 0.54 & 0.8 & 0.82 & $-48 / 52$ \\
\hline
\end{tabular}

${ }^{1}$ Data from Akagi et al., 2011 

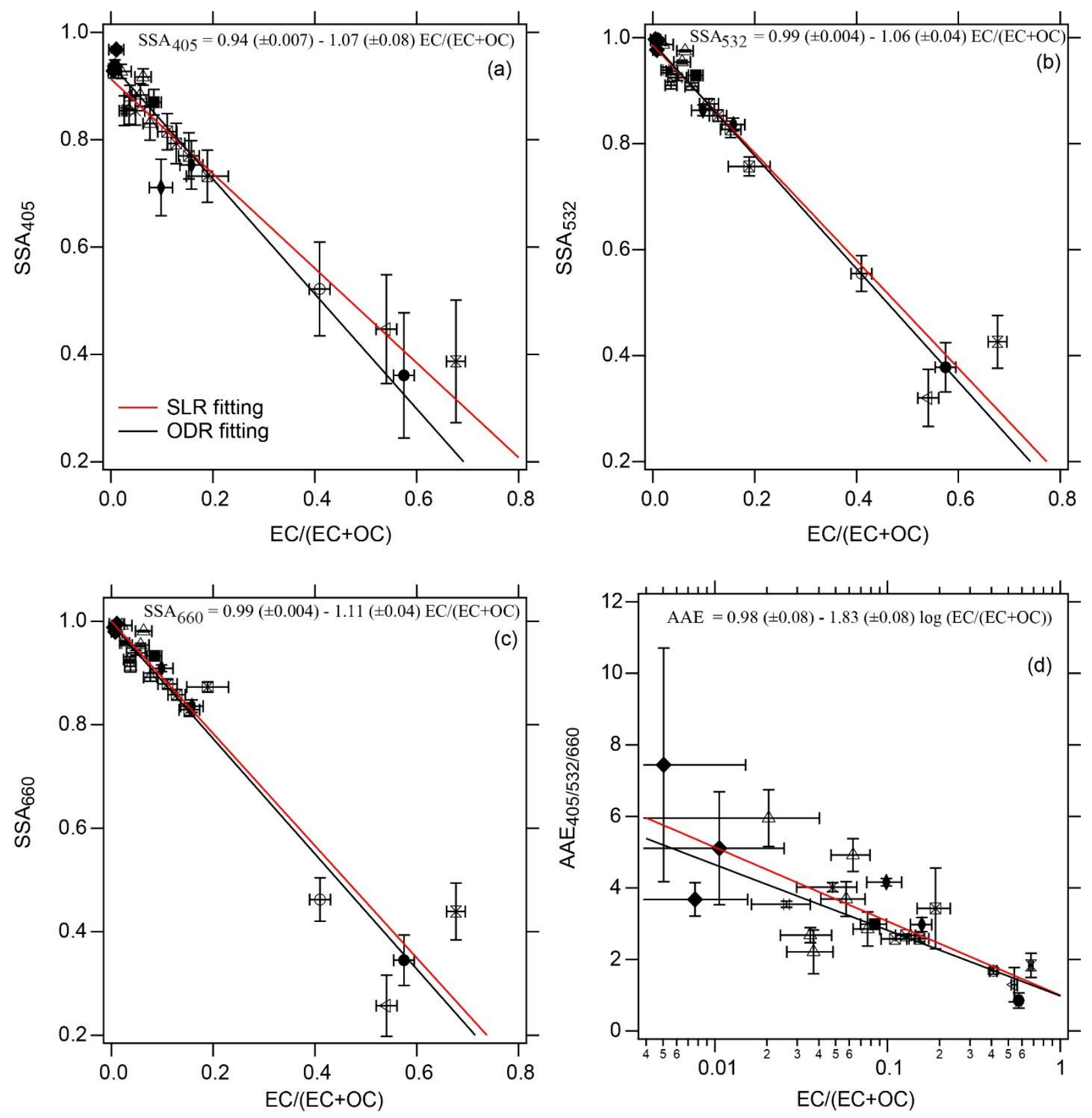

Figure S1. SSA at (a) $405 \mathrm{~nm}$, (b) $532 \mathrm{~nm}$, (c) $660 \mathrm{~nm}$, and (d) AAE plotted as a function of EC/(EC+OC). Solid red lines are simple linear regression fitting lines and solid black lines are orthogonal distance regression fitting lines. Equations presented in each figures are the ODR best fit equations for respective SSA and AAE. 


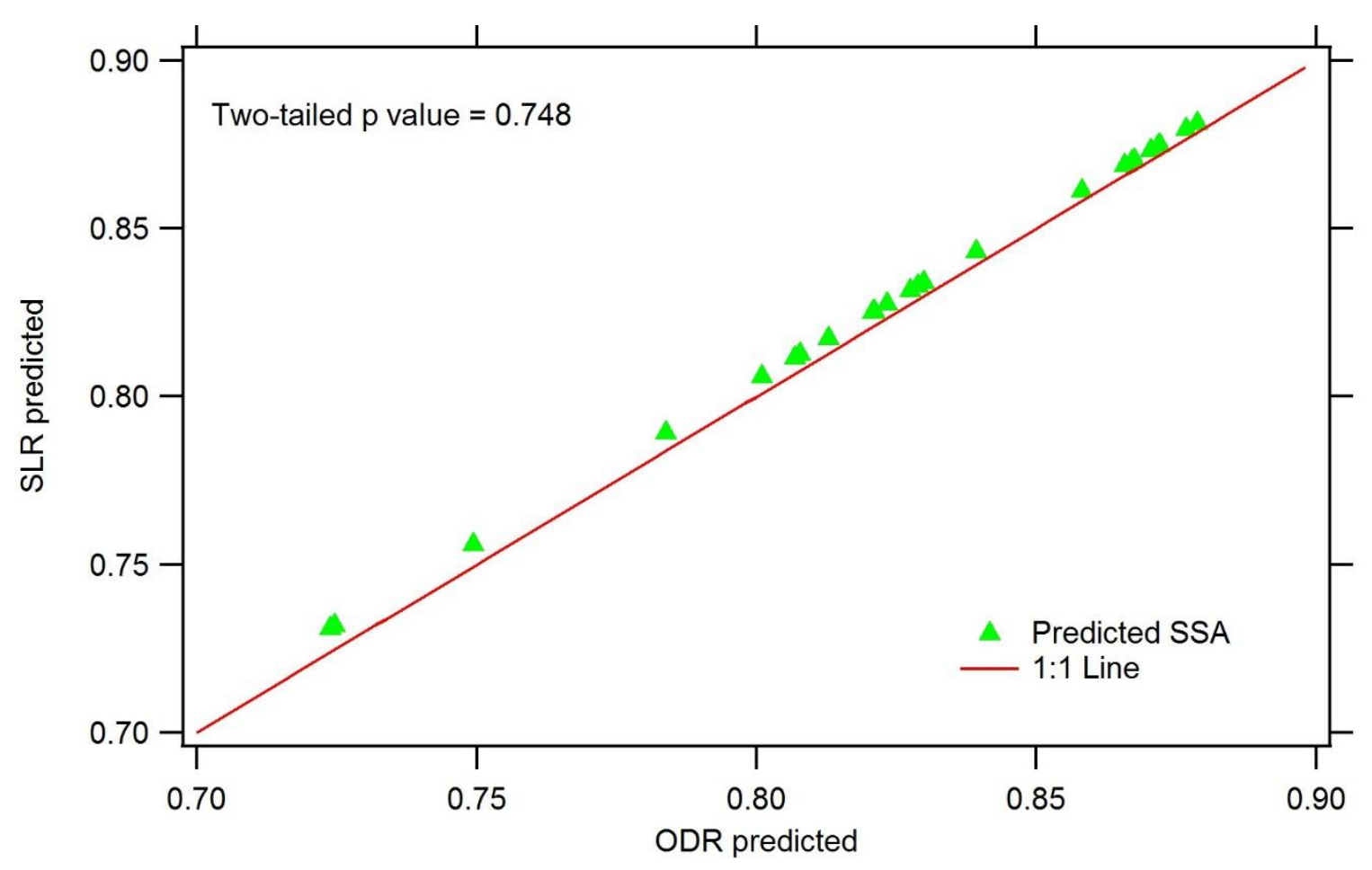

Figure S2. Comparison of predicted SSA values based on SLR and ODR models. Solid red line is one to one line. 